\title{
LINEAMIENTOS TEÓRICOS Y METODOLÓGICOS DE LA INVESTIGACIÓN CUANTITATIVA EN CIENCIAS SOCIALES
}

\section{Theoretical And Methodological Guidelines of the Quantitative Research in Social Sciences}

\author{
Elmina Matilde Rivadeneira Rodríguez*
}

\begin{abstract}
RESUMEN
T a presente investigación tiene por objetivo analizar los lineamientos teóricos para Lcomprender el proceso metodológico de la investigación cuantitativa en las ciencias sociales. Los métodos cuantitativos estudian los hechos observables, medibles y replicables donde se utiliza con precisión matemática y con modelos estadísticos de codificación numérica. La investigación cuantitativa destaca las siguientes características importantes como: problema, objetivo general y específicos, hipótesis, variables, instrumentos para la recolección de la información y medición de variables muy estructuradas. En las ciencias humanas y sociales en las últimas décadas han abordado una participación en la investigación, en donde la metodología cuantitativa y cualitativa estudia el fenómeno.
\end{abstract}

Palabras clave: medible, metodológico, teórico

\begin{abstract}
The present research aims to analyze the theoretical guidelines to understand the methodological process of quantitative research in the social sciences. Quantitative methods study the observable, measurable and replicable facts where it is used with mathematical precision and with statistical models of numerical coding. Quantitative research highlights the following important characteristics: problem, general and specific objective, hypotheses, variables, instruments for the collection of information and measurement of highly structured variables. In the human and social sciences in the last decades they have approached a participation in the research, where the quantitative and qualitative methodology studies the phenomenon
\end{abstract}

Keywords: Measurable, Methodological, Theoretical,

* Universidad Nacional de Educación, Ecuador. Correo electrónico: elminar@hotmail.com 


\section{INTRODUCCIÓN}

El ser humano tiene como finalidad de vida buscar, indagar para darle sentido a las cosas. De esto se deduce que existen diferentes tipos de investigaciones, desde las más elementales y cotidianas por las cuales se busca ampliar el horizonte de los objetos conocidos, hasta la investigación científica con características propias de eficiencia superior. La investigación social cuantitativa está relacionado con el paradigma explicativo. Este paradigma utiliza preferentemente información cuantificada para poder explicar los fenómenos

Si bien es cierto que los conocimientos teóricos son fundamentales para la praxis investigativa, no debemos considerarlos suficientes por sí mismos. Y esto porque investigar es una práctica que retorna sobre sí misma, que se apropia de las operaciones, acciones y caminos recorridos, y utiliza sus errores para reformularse.

\section{PROBLEMA}

Al desarrollar la investigación se pretende responder la siguiente interrogante principal:

¿Cuáles con los lineamientos teóricos y metodológicos que permiten comprender el proceso de la investigación cuantitativa social?

Esto conlleva a responder las siguientes interrogantes:

- ¿Cuáles son los lineamientos teóricos que permiten el proceso de explicación de la investigación cuantitativa social?

- ¿Cuáles son los lineamientos metodológicos que permiten desarrollar la metodología de la investigación cuantitativa social?

- ¿Cuáles son los lineamientos teóricos y metodológicos que permitan el proceso de la investigación cuantitativa social?

\section{Objetivo general}

Analizar los lineamientos teóricos y metodológicos de la investigación cuantitativa social para comprender el proceso de la investigación.

\section{Objetivos específicos}

- Analizar los lineamientos teóricos para garantizar la explicación de la investigación cuantitativa social.

- Analizar los lineamientos metodológicos para garantizar el proceso de la investigación cuantitativa social.

- Describir los lineamientos teóricos y metodológicos que permitan la explicación y el proceso de la investigación cuantitativa social. 


\section{METODOLOGÍA}

La metodología es eminentemente teórica se basó en una investigación bibliográfica porque su objetivo es analizar los teóricos y metodológicos para comprender el proceso de la investigación cuantitativa social. Respecto a su diseño es de tipo documental, en esta investigación se utilizó la técnica de análisis de contenido, aplicado a discursos, información, mensajes, textos, imágenes.

\section{REVISIÓN TEÓRICA}

La investigación cuantitativa parte de un paradigma positivista, cuyo propósito es describir y explicar casualmente, así como generalizar extrapolar y universalizar, siendo el objeto de esta investigación hechos, objetivos existentes y sometidos a leyes y patrones generales. Esta investigación recoge y analiza datos sobre variables y estudia las propiedades y fenómenos cuantitativos, es decir sus objetivos son cuantitativos.

Este tipo de investigación estudia hechos observables, medibles y replicables, haciéndolo en un contexto controlado donde se utiliza un lenguaje con precisión matemática y con modelos estadísticos de la codificación numérica.

La investigación cuantitativa destaca que las características importantes se pueden mencionar:

- Problema bien definido por el investigador.

- Los objetivos claramente definidos por el investigador

- Se elaboran hipótesis para ser verificados o falseados mediante pruebas empíricas.

- Utiliza instrumentos para recolección de información y medición de variables muy estructuradas.

\section{EL PARADIGMA MODERNISTA}

La Modernidad determina sus premisas fundamentales: como principio didáctico, la Ilustración Europea - Iluminismo Francés - hace su aparición para educar, esto es cohibir de la mejor manera posible, los instintos y exaltar, hasta la sublimación, la ejecución de conductas y convenciones sociales es previamente establecidas. El segundo principio se sustenta en el positivismo, corriente ideológica cuyo cauce esencial sigue el curso de una filosofía progresista, civilizatoria, dentro de la cual el ser humano es valorado en razón de su inserción al progreso productivo industrial y al esquema de organización social por él y para él previsto. Desde esta perspectiva, la Modernidad sentó las bases para una existencia estabilizada, plena de confort. El discurso científico obedecerá a una racionalidad formal, a esto se conoce como el modelo epistémico o paradigma conocido también como el positivismo. El positivismo es un conjunto de reglamentaciones que rigen el saber humano. A través de la historia ha realizado críticas contra los desarrollos metafísicos (Kolakowski, 1966). Además se caracteriza por sostener que el conocimiento verdadero es aquel pro- 
ducido por la ciencia y asume un método específico para conocer la realidad (Dobles, Zúñiga y García, 1998), los positivistas buscan los hechos o causas de los de fenómenos sociales con independencia de los estados subjetivos de los individuos (Gutiérrez, 1996)

Para Martínez (1999) el paradigma epistemológico positivista de las ciencias sociales tomó el modelo físico de Newton para poder concebir la realidad, la racionalidad de Descartes y la metodología inductiva de Bacón. Investigadores que valoran la objetividad del conocimiento, el de los fenómenos, la experiencia sensible, la cuantificación aleatoria determinismo de las medidas, la verificación empírica la demostración experimental. La investigación cuantitativa tiene una concepción lineal, es decir que exista claridad entre los elementos que conforman el problema (Hurtado y Toro, 1998)

\section{METODOLOGÍA CUANTITATIVA}

Figura 1. Partes del método científico

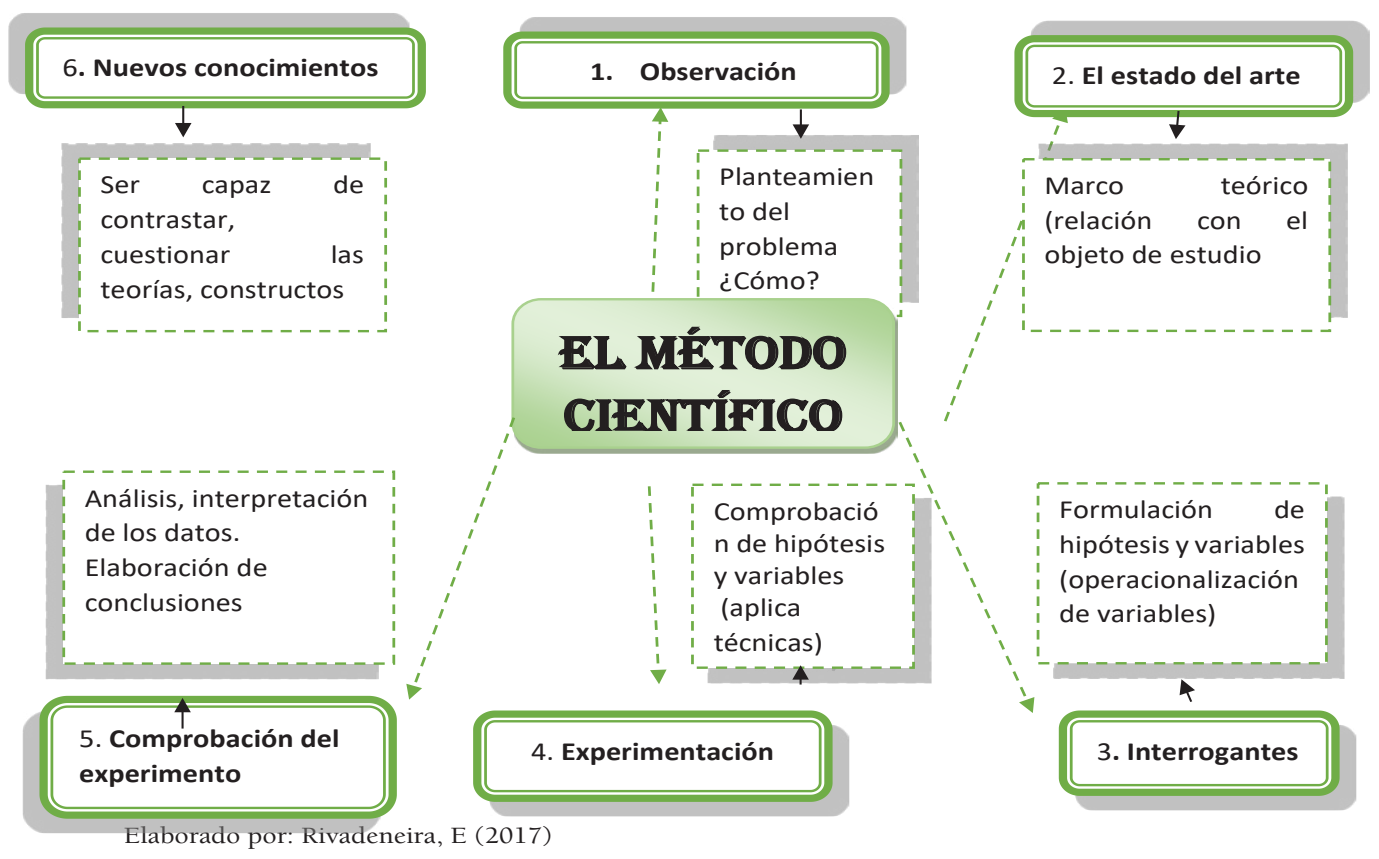

Partes del diseño de la metodología cuantitativa:

- Elección del tema

- Selección del problema

- Objetivos de la investigación: general y específicos

- Alcances y limitaciones

- Hipótesis

- Variables

- Metodología 
- Población y muestra

- Técnicas de investigación

- Marco teórico

- Instrumentos para la recolección de datos

- Validez y confiabilidad del instrumento: objetivos, variables, indicadores

- Análisis de datos: tabulación de datos, tratamiento de datos, interpretación de datos

- Conclusiones y recomendaciones

- Referencias bibliográficas

Se puede definir como un conjunto de procedimientos o reglas generales por medio de las cuales se investiga el objeto de estudio de la ciencia.

Para Kuprian (1978) el método es la cadena ordenada de pasos (acciones) basada en un aparato conceptual de reglas que permiten avanzar en el proceso del conocimiento, desde lo conocido a lo desconocido.

El método científico es el procedimiento planteado que se sigue en la investigación para descubrir las formas de existencia de los procesos objetivos, es decir para explorar, describir, explicar, correlacionar sus conexiones internas y externas, para poder generalizar y profundizar los conocimientos adquiridos, para poder llegar a demostrarlos con rigor racional y para comprobarlos en el experimento y con las técnicas aplicada

Rodríguez (2010) afirma que el método cuantitativo se centra en los hechos o causas del fenómeno social, Este método utiliza el cuestionario, inventarios y análisis demográficos que producen números, los cuales pueden ser analizados estadísticamente para verificar, aprobar o rechazar las relaciones entre las variables definidas operacionalmente, además regularmente la presentación de resultados de estudios cuantitativos viene sustentada con tablas estadísticas, gráficas y un análisis numérico.

\section{ENFOQUE CUANTITATIVO}

En el marco de los distintos grados de medición, manipulación y control, se distinguen tres modalidades metodológicas en el enfoque cuantitativo: experimental, cuasi experimental y no experimental.

El diseño experimental tiene que ver con la manipulación de variables. El diseño cuasiexperimental es aquel que tiene la imposibilidad de manipular la variable independiente. En el diseño no experimental no se puede manejar las variables que interfieren en el estudio 
Tabla 1. Enfoque cuantitativo

- $\quad$ Con perspectiva de cientificista

- Se apoya en las teorías

- Énfasis en la explicación, en la contratación empírica y en la medición objetiva de fenómenos

- Propensión a servirse de los sujetos de estudio

- Se limita a responder

- Son débiles en términos de validez interna - casi nunca sabemos si se miden lo que quieren medir - pero son fuertes en validez externa, lo que encuentran es generalizable a la población

- Abordan aspectos estables de la realidad

- Se basan en una muestra probabilística

- Criterios de fiabilidad

- Se apoya en un paradigma racionalista hipotético-deductivo, propio del positivismo lógico. Perspectiva analítica-positivista

- Se gana en precisión

- Facilitan la comparación, pero tiene limitaciones para hacer matizaciones

- $\quad$ Estandarizadas, pero sin matices

- Preguntan a los cualitativos: ¿Son generalizables tus hallazgos

- Usa recolección de datos para probar la hipótesis con base en la medición numérica y el análisis estadístico para establecer patrones de comportamiento

Tabla 2. Comparación entre enfoque cuantitativo y cualitativo

\begin{tabular}{llll}
\hline \multirow{2}{*}{ ASPECTOS } & \multicolumn{1}{c}{ Enfoque cuantitativo } & \multicolumn{2}{c}{ Enfoque cualitativo } \\
\cline { 2 - 4 } $\begin{array}{l}\text { Positivista } \\
\text { Problidad }\end{array}$ & \multicolumn{1}{c}{ Simple, fragmentado } & Múltiple, divergente & \multicolumn{1}{c}{ Crítico } \\
& Surgen de teorías & $\begin{array}{l}\text { Los grupos sociales origi- } \\
\text { nan el problema }\end{array}$ & $\begin{array}{l}\text { Parten de las situaciones } \\
\text { reales para transformar }\end{array}$ \\
Diseño & Estructura & Abierto y flexible & Diálogo, consenso \\
Muestra & Grande, representativa & Pequeña, reajustable & No es de interés la represen- \\
& & & tación \\
Tipo de análisis & $\begin{array}{l}\text { Análisis causal-correla- } \\
\text { cional }\end{array}$ & $\begin{array}{l}\text { Análisis descriptivo-inter- } \\
\text { pretativo }\end{array}$ & $\begin{array}{l}\text { Análisis crítico-transforma- } \\
\text { dor }\end{array}$
\end{tabular}

Elaborado por: Rivadeneira (2012)

\section{CONCLUSIONES}

Una vez efectuada la revisión de los diferentes planteamientos teóricos para cumplir con los objetivos que guían la presente investigación se enuncian las siguientes conclusiones:

El objetivo general es: "Analizar los lineamientos teóricos y metodológicos de la investigación cuantitativa social para comprender el proceso de la investigación". El desarrollo de este objetivo se realiza mediante tres objetivos específicos:

- Objetivo específico 1. "Analizar los lineamientos teóricos para garantizar la explicación de la investigación cuantitativa social". Esta investigación estudia hechos 
observables, medibles y replicables, haciéndolo en un contexto controlado donde se utiliza un lenguaje con precisión matemática y estadística. El paradigma positivista crítica y rechaza los desarrollos metafísicos.

- Objetivo específico 2. "Analizar los lineamientos metodológicos para garantizar el proceso de la investigación cuantitativa social”. En esta investigación el diseño metodológico es rígido, sus características más relevantes son que es objetivo, producto, método deductivo, externa y básicamente en el diseño no puede faltar la hipótesis.

- Objetivo específico 3. "Describir los lineamientos teóricos y metodológicos que permitan la explicación y el proceso de la investigación cuantitativa social". El estudio de los problemas, hechos, acontecimientos, fenómenos, es decir, el investigador sabe de antemano lo que está buscando, todos los aspectos de estudio son diseñados antes de la recogida de datos, el investigador utiliza herramientas tales como cuestionarios o equipos para recoger datos numéricos.

\section{REFERENCIAS BIBLIOGRÁFICAS}

Dobles, C., Zúñiga, M. y García, J. (1998). Investigación en educación: procesos, interacciones y construcciones. San José: EUNED.

Gutiérrez, L. (1996). Paradigmas cuantitativo y cualitativo en la investigación socio-educativa: proyecciones y reflexiones. Revista PARADIGMA. Volumen XIV al XVII

Hurtado, I. y Toro, J. (1998): Paradigmas y métodos de investigación en tiempos de cambio. Segunda edición. Ediciones de la Universidad de Carabobo. Venezuela

Kolakowski, L. (1966). La filosofía positivista. Ediciones Cátedra. Madrid

Kuprian, A. (1978). Problemas metodológicos del experimento social. Editorial de Ciencias Sociales. La Habana

Martínez, M. (1999). La Nueva Ciencia. Su desafío, Lógica y Método. Primera Edición México

Rodríguez, A. (2010). Métodos de investigación. México: Universidad Autónoma de Sinaloa. 